\title{
CFD-BASED OPTIMIZATION OF ROTOR ELECTRO-THERMAL ICE PROTECTION SYSTEMS
}

\author{
Adam Targui ${ }^{*}$, Wagdi G. Habashi ${ }^{\dagger}$ \\ CFD Laboratory, Department of Mechanical Engineering, McGill University, Montreal, Canada \\ *adam.targui@cfdlab.mcgill.ca, †wagdi.habashi@mcgill.ca
}

\begin{abstract}
The helicopter rotor is responsible for lift generation and control along the pitch and roll axes and is therefore an essential component to protect against ice accretion, a hazardous phenomenon that can lead to departure from controlled flight. Ice protection systems (IPS) used in helicopters differ from that of aircraft due to the smaller wing cross-section and the lower onboard power available. Electro-thermal heating pads are a prevalent solution answering these constraints, as they are thin and can fully conform to a blade profile. Current research to optimize electro-thermal IPS is limited to airfoils, while flows and icing on aircraft wings and helicopter rotors are highly three-dimensional in nature. The present methodology extends IPS optimization to include electro-thermal IPS for three-dimensional wings as well as rotorcraft in hover and forward flight.
\end{abstract}

The governing physics are those of a conjugate heat transfer problem between a fluid and a solid domain. The flow solution is obtained by solving the steady Reynolds-Averaged Navier-Stokes (RANS) equations. In the solid domain, heat generation by the electric pads and thermal conduction through the blade affect the heat flux to the external flow. Furthermore, the energy balance at their interface must account for icing. Simulation results are provided by the FENSAP-ICE system, augmented with compatible tools.

Evaluating a CFD-based objective function requires running computationally expensive flow and heat transfer simulations. Repeating this operation every iteration would make this problem intractable. Therefore, a Reduced Order Model (ROM) is built to return the objective function value to the optimizer at each iteration, making the robust derivative-free optimizer NOMAD a natural choice for this study.

The approach provides the user with a selection of problem formulations with various design variables and objective functions. Using the geometry of the Caradonna-Tung experiments the methodology has been applied to minimize lift degradation for a hovering rotor by optimizing heater power distribution under the constraint of maximum power availability. It is found that the optimal distribution can yield lift improvements of $7 \%$ and torque of $10 \%$, highlighting the importance of adequate power distribution for the IPS. 